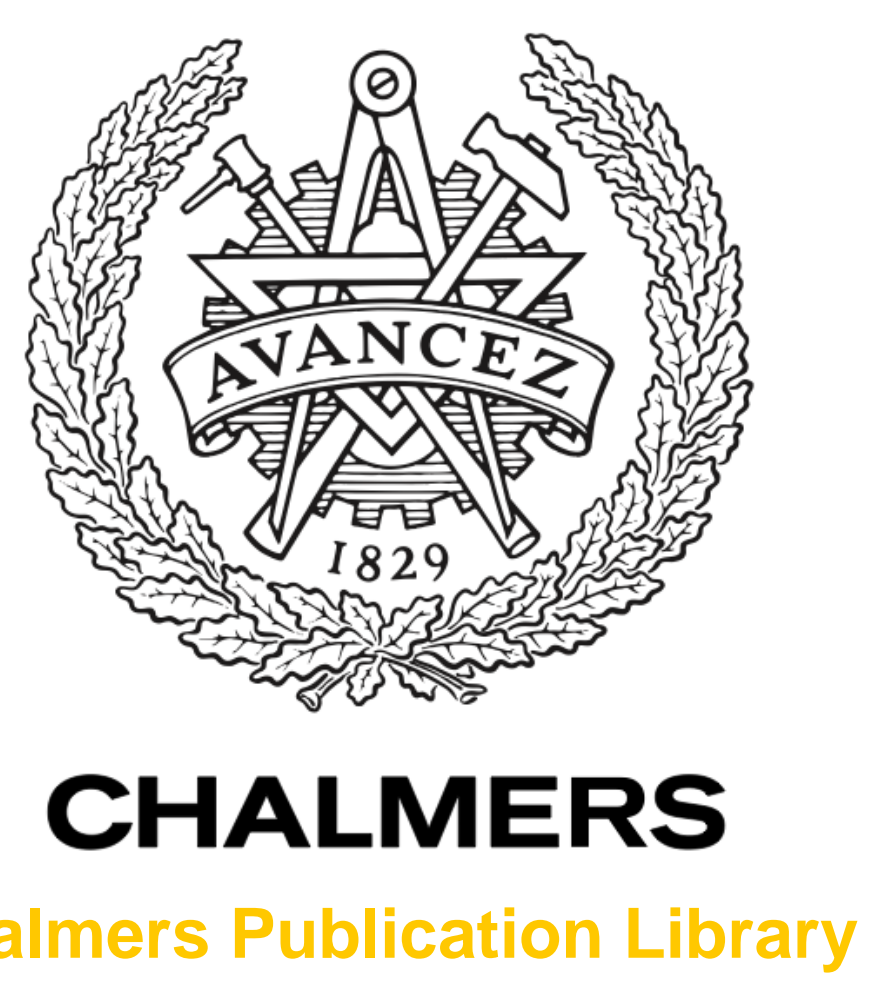

Chalmers Publication Library

\title{
Calibration of indoor UWB sub-band divided ray tracing using multiobjective simulated annealing
}

This document has been downloaded from Chalmers Publication Library $(\mathrm{CPL})$. It is the author's version of a work that was accepted for publication in:

2014 IEEE International Conference on Communications, ICC 2014

\author{
Citation for the published paper: \\ Gan, M. ; Meissner, P. ; Mani, F. (2014) "Calibration of indoor UWB sub-band divided ray \\ tracing using multiobjective simulated annealing". 2014 IEEE International Conference on \\ Communications, ICC 2014 pp. 4844-4849.
}

http://dx.doi.org/10.1109/ICC.2014.6884087

Downloaded from: http://publications.lib.chalmers.se/publication/203318

Notice: Changes introduced as a result of publishing processes such as copy-editing and formatting may not be reflected in this document. For a definitive version of this work, please refer to the published source. Please note that access to the published version might require a subscription.

Chalmers Publication Library (CPL) offers the possibility of retrieving research publications produced at Chalmers University of Technology. It covers all types of publications: articles, dissertations, licentiate theses, masters theses, conference papers, reports etc. Since 2006 it is the official tool for Chalmers official publication statistics. To ensure that Chalmers research results are disseminated as widely as possible, an Open Access Policy has been adopted.

The CPL service is administrated and maintained by Chalmers Library. 


\title{
Calibration of Indoor UWB Sub-band Divided Ray Tracing Using Multiobjective Simulated Annealing
}

\author{
Mingming Gan ${ }^{1}$, Paul Meissner ${ }^{2}$, Francesco Mani ${ }^{3}$, Erik Leitinger ${ }^{2}$, \\ Markus Fröhle $^{4}$, Claude Oestges ${ }^{5}$, Klaus Witrisal ${ }^{2}$, Thomas Zemen ${ }^{1}$ \\ ${ }^{1}$ FTW Forschungszentrum Telekommunikation Wien, Austria; ${ }^{2}$ Graz University of Technology, Austria; \\ ${ }^{3}$ COMELEC Department, Telecom ParisTech, France; ${ }^{4}$ Chalmers University of Technology, Sweden; \\ ${ }^{5}$ ICTEAM, Université catholique de Louvain, Belgium
}

\begin{abstract}
Sub-band divided ray tracing (RT) has been widely used to reproduce as reliably as possible the ultra-wideband (UWB) radio wave propagation channel in realistic indoor environments. However, its accuracy is strictly limited by the available description of the environment. Moreover, its computational complexity scales with the number of selected subbands and the number of propagation paths. In the present work, our RT tool considers not only deterministic propagation paths but also diffuse scattering components. Based on a low-complexity sub-band divided RT implementation, we propose a calibration method for indoor UWB sub-band divided RT. The method estimates the optimal material parameters, including the dielectric parameters and the scattering parameters, using channel measurements and multiobjective simulated annealing (MOSA). This calibration can improve the accuracy of sub-band divided RT in terms of the power delay profile (PDP) and the root mean square (RMS) delay spread for all test locations including those not considered by the calibration. A measurement campaign is used to verify the calibration technique.
\end{abstract}

Keywords - ray tracing (RT), low-complexity, ultra-wideband (UWB), Multiobjective simulated annealing (MOSA).

\section{INTRODUCTION}

In recent years, UWB technology has gained a large amount attention and has been applied in many fields. Deep understanding of its channel characteristics is crucial for tasks, such as indoor localization and tracking [1], [2]. For UWB system, the channel characteristics vary significantly within the entire bandwidth. Deterministic sub-band divided RT technique divides the frequency range of interest into multiple subbands. RT is performed at the center frequencies of every subband. Finally all sub-band frequency responses are combined to obtain the frequency response for the full UWB bandwidth.

It is known that the accuracy of RT is related to the exact knowledge of all material parameters describing the

The author would like to thank Miss Xuhong $\mathrm{Li}$ for assistance with speeding up the ray tracing simulation. The work of M. Gan and T. Zemen is supported by the Austrian Science Fund (FWF) through grant NFN SISE (S10607) and by the Austrian Competence Center FTW Forschungszentrum Telekommunikation Wien $\mathrm{GmbH}$ within project I0. FTW is funded within the program COMET - Competence Centers for Excellent Technologies by BMVIT, BMWFJ, and the City of Vienna. The COMET program is managed by the FFG. The work of P. Meissner, E. Leitinger and K. Witrisal was partly supported by the Austrian Science Fund (FWF) within the National Research Network SISE (S10610), and by the Austria Research Promotion Agency (FFG) within KIRAS PL3, grant nb. 832335 "LOBSTER". This collaboration was also part of the COST action IC1004 entitled "Cooperative Radio Communications for Green Smart Environments". scattering environment. Although the reflection and transmission properties of building materials can be measured at UWB frequencies, the measured dielectric values can be inhomogeneous even in the same environment because of changing temperature and humidity conditions. Therefore, it is impossible to accurately define these dielectric parameters for each building. The situation is further exacerbated when the materials are made of a mixture of unknown components. Moreover, our RT tool involves not only the specular paths but also the diffuse scattering paths. The parameters used for the scattering mechanism cannot be directly measured. Hence, the diffuse scattering model parameters must be fitted by comparing to empirical measurement data for different materials [3].

For all these reasons the approximation of the input material parameters lead to a mismatch between the simulation results of RT and empiric measurements [4]. Therefore, it is necessary to implement a calibration algorithm for these RT parameters to improve the prediction accuracy of RT. To date, the calibration of RT based on the single objective optimization has been presented, but the diffuse scattering components are not considered in [5], [6]. Due to the nonlinear combinatorial relationship between the power taps and material dielectric parameters, a simulated annealing approach is used in these works. This method provides a general optimal solution by simultaneously tuning the permittivity and conductivity of all materials to optimize the defined objective function.

In order to significantly improve the accuracy of sub-band divided RT for the UWB indoor channels, the intensively computational simulation time need to be reduced first. This work has been done by a low-complexity sub-band divided RT, which means not only the geometric calculation but also the electromagnetic calculation needs to be performed once for all subbands [7]. The main contribution of this paper are the following:

- MOSA is a calibration algorithm to optimize the input material parameters within a given range, which refers to the dielectric and scattering parameters. The weighted sum approach is used for the proposed MOSA by projecting the multidimensional objective space into a single space.

- We separate the measurements into specular and diffuse scattering components by cross correlation and a search 
and subtract algorithm.

- The accuracy of the calibrated low-complexity sub-band divided RT algorithm is verified through measurements. Even though only one pilot measurement is used for the calibration, a significant improvement of the accuracy is obtained.

\section{LOW-COMPLEXITY SUB-BAND DiVIDED RT}

\section{A. RT Tool}

Our RT tool is three-dimensional (3D) and the considered propagation mechanisms are line-of-sight (LOS), reflection, penetration, diffraction and diffuse scattering. Two core calculations are: (i) the geometric computation and (ii) the electromagnetic computation [8]. The propagation paths relevant at a given location can be obtained based on the geometric computation, which is related to the accurate description of the 3D environmental database and optical principles. The electromagnetic computation calculates the electric field of the propagation path in amplitude, phase and polarization relying upon the relevant propagation mechanisms and the antenna radiation patterns [9].

A detailed description of the $3 \mathrm{D}$ environment is required, not only dimensions of all blocks, such as walls, windows, doors, but also the material properties including the dielectric permittivity $\varepsilon_{r}$ and conductivity $\sigma$, the scattering coefficient $S$ and the integer $\alpha$ indicating the width of the scattering lobe. These parameters are significant for propagation mechanisms accounted in the RT tool [7], but getting the accurate values of these parameters is very difficult. In [3], some numerical results have shown that different $S$ and $\alpha$ values can greatly influence the power gain of the diffuse scattering components.

The complex dyadic reflection and penetration coefficients involved in the corresponding propagation mechanisms are obtained by Fresnel formulas, whereas diffraction coefficients are calculated by the uniform theory of diffraction (UTD) [9]. The reflection reduction factor $R$ indicates the amount of energy by which perfect specular reflections are reduced due to diffuse scattering and is defined as [10]:

$$
R \cong \sqrt{1-S^{2}}
$$

The path direction is not modified by the penetration mechanism in our RT tool. Moreover, the penetration case is embedded into all other propagation mechanisms.

It is known that a flat wave is scattered into multiple random directions when it is interacting with a rough surface. This mechanism has been implemented in our tool based on a directive scattering model, which assumes that the scattering lobe related to the scattering tile is steered towards the direction of the specular reflection [3]. This scattering tile is determined by recursively dividing the surface until the far field condition is fulfilled [11]. The amplitude of the diffuse scattering field is evaluated based on this model, and then a uniformly distributed random phase is added to each diffuse scattering path due to the incoherent property of the scattering paths.

\section{B. Low-complexity Sub-band Divided RT}

The sub-band divided RT algorithm applied to UWB radio channels has been presented in [12], [13]. The entire UWB bandwidth $B$ is divided into $i$ equal subbands with bandwidth $B_{i}$, where $i \in\{1, \cdots, I\}$ and $I$ is the total number of the subands, and $B_{i} \leq 500 \mathrm{MHz}$. Instead of implementing RT at one discrete frequency point as for narrowband systems, subband divided RT has to be performed at multiple frequencies $f_{\mathrm{c}, i}$, which are the respective center frequencies of each subband. The computational complexity of the sub-band divided $\mathrm{RT}$ at one receiver $(\mathrm{Rx})$ location is directly proportional to the number of subbands.

Low-complexity sub-band divided RT is introduced in [7], the basic idea of [7] is that not only the geometric computation but also the electromagnetic computation only needs to be performed once at the center frequency $f_{\mathrm{c}, i}$ of one subband $i$. Then the corresponding calculation for other subbands can be derived directly according to the physical properties of the propagation mechanisms. It is worth mentioning that distinguishing how the dielectric properties vary with frequencies is very difficult, so that it is assumed that the dielectric properties $\varepsilon_{r}$ and $\sigma$ do not depend on the frequency within the entire UWB bandwidth [4], [14]. With the complex electric field $\eta_{\{\cdot\}}\left(f_{\mathrm{c}, i}\right)$ of the propagation path at the center frequency $f_{\mathrm{c}, i}$ of the $i$-th subband is obtained, the resulting complex electric field $\eta_{\{\cdot\}}\left(f_{\mathrm{c}, i^{\prime}}\right)$ at all other center frequency $f_{\mathrm{c}, i^{\prime}}$ can be calculated [7]. Here, we take the single order propagation case as an example:

- LOS component:

$$
\begin{aligned}
\eta_{\mathrm{RT}_{\mathrm{LOS}}}\left(f_{\mathrm{c}, i^{\prime}}\right)= & \eta_{\mathrm{RT}_{\mathrm{LOS}}}\left(f_{\mathrm{c}, i}\right) \cdot\left[\frac{\bar{g}_{\mathrm{LOS}}^{\mathrm{R}}\left(f_{\mathrm{c}, i^{\prime}}\right)}{\bar{g}_{\mathrm{LOS}}^{\mathrm{R}}\left(f_{\mathrm{c}, i}\right)}\right]^{*} \cdot \\
& \frac{f_{\mathrm{c}, i}}{f_{\mathrm{c}, i^{\prime}}} \cdot\left[\frac{\bar{g}_{\mathrm{LOS}}^{\mathrm{E}}\left(f_{\mathrm{c}, i^{\prime}}\right)}{\bar{g}_{\mathrm{LOS}}^{\mathrm{E}}\left(f_{\mathrm{c}, i}\right)}\right] e^{\frac{-j 2 \pi\left(f_{\mathrm{c}, i^{\prime}}-f_{\mathrm{c}, i}\right) s}{c}},
\end{aligned}
$$

- Reflection/penetration component:

$$
\begin{aligned}
& \eta_{\mathrm{RT}_{\mathrm{r} / \mathrm{p}}}\left(f_{\mathrm{c}, i^{\prime}}\right)=\eta_{\mathrm{RT}_{\mathrm{r} / \mathrm{p}}}\left(f_{\mathrm{c}, i}\right) \cdot\left[\frac{\bar{g}_{\mathrm{r} / \mathrm{p}}^{\mathrm{R}}\left(f_{\mathrm{c}, i^{\prime}}\right)}{\bar{g}_{\mathrm{r} / \mathrm{p}}^{\mathrm{R}}\left(f_{\mathrm{c}, i}\right)}\right]^{*} . \\
& \frac{f_{\mathrm{c}, i}}{f_{\mathrm{c}, i^{\prime}}} \cdot\left[\frac{\bar{g}_{\mathrm{r} / \mathrm{p}}^{\mathrm{E}}\left(f_{\mathrm{c}, i^{\prime}}\right)}{\bar{g}_{\mathrm{r} / \mathrm{p}}^{\mathrm{E}}\left(f_{\mathrm{c}, i}\right)}\right] e^{\frac{-j 2 \pi\left(f_{\mathrm{c}, i^{\prime}}-f_{\mathrm{c}, i}\right) s}{c}},
\end{aligned}
$$

- Diffraction component:

$$
\begin{aligned}
\eta_{\mathrm{RT}_{\mathrm{d}}}\left(f_{\mathrm{c}, i^{\prime}}\right)= & \eta_{\mathrm{RT}_{\mathrm{d}}}\left(f_{\mathrm{c}, i}\right) \cdot\left[\frac{\bar{g}_{\mathrm{d}}^{\mathrm{R}}\left(f_{\mathrm{c}, i^{\prime}}\right)}{\bar{g}_{\mathrm{d}}^{\mathrm{R}}\left(f_{\mathrm{c}, i}\right)}\right]^{*} \cdot \\
& \left(\frac{f_{\mathrm{c}, i}}{f_{\mathrm{c}, i^{\prime}}}\right)^{\frac{3}{2}} \cdot\left[\frac{\bar{g}_{\mathrm{d}}^{\mathrm{E}}\left(f_{\mathrm{c}, i^{\prime}}\right)}{\bar{g}_{\mathrm{d}}^{\mathrm{E}}\left(f_{\mathrm{c}, i}\right)}\right] e^{\frac{-j 2 \pi\left(f_{\mathrm{c}, i^{\prime}}-f_{\mathrm{c}, i}\right) s}{c}},
\end{aligned}
$$

- Diffuse scattering component:

$$
\eta_{\mathrm{RT}_{\mathrm{s}}}\left(f_{\mathrm{c}, i^{\prime}}\right)=\eta_{\mathrm{RT}_{\mathrm{s}}}\left(f_{\mathrm{c}, i}\right) \cdot\left[\frac{\bar{g}_{\mathrm{s}}^{\mathrm{R}}\left(f_{\mathrm{c}, i^{\prime}}\right)}{\bar{g}_{\mathrm{s}}^{\mathrm{R}}\left(f_{\mathrm{c}, i}\right)}\right]^{*} \cdot \frac{f_{\mathrm{c}, i}}{f_{\mathrm{c}, i^{\prime}}} \cdot\left[\frac{\bar{g}_{\mathrm{s}}^{\mathrm{E}}\left(f_{\mathrm{c}, i^{\prime}}\right)}{\bar{g}_{\mathrm{s}}^{\mathrm{E}}\left(f_{\mathrm{c}, i}\right)}\right] e^{-j \theta_{\mathrm{s}}^{\prime}},
$$

where $s$ is the total distance between transmitter (Tx) and Rx, $c$ is the speed of light, $\theta_{\mathrm{s}}^{\prime}$ indicates the random phase with an uniform distribution in $[0,2 \pi] . \bar{g}_{\{\cdot\}}^{\mathrm{E}}\left(f_{\mathrm{c}, i}\right)$ and $\bar{g}_{\{\cdot\}}^{\mathrm{R}}\left(f_{\mathrm{c}, i}\right)$ are the complex vectors accounting for the $\mathrm{Tx} / \mathrm{Rx}$ antenna polarization 
and amplitude gains within one subband in the direction of the propagation wave. For brevity, we omit the arguments of the azimuth and elevation directions of the transmitted/received wave, because the geometrical calculation of each propagation path is identical at different subbands for one $\operatorname{Rx}$ location. $\{\cdot\}^{*}$ designates the complex conjugate. The detailed information can be found in [7].

\section{Channel Model Calibration}

Simulated annealing (SA) for single objective optimization has already been employed for RT calibration without taking diffuse scattering components into account [5], [12]. Based on our RT tool, we propose to optimize not only $\varepsilon_{r}$ and $\sigma$ but also $S$ and $\alpha$ for each material, which play an important role for the propagation mechanisms. It has been mentioned that the reflection component and the scattering components intimately interact with each other, so that multiobjective optimization has to be considered.

\section{A. Distinguish Deterministic and Diffuse Scattering Compo- nents in Measurements}

The search and subtract approach is a maximum likelihood method for separating the deterministic and diffuse scattering paths of CIR measurements [15]. The measurement data is available in the frequency domain, so the CTF at $N_{\mathrm{f}}$ frequency points for one Rx's location is expressed as

$$
\mathbf{H}_{\text {Meas }}=\left[H_{\text {Meas }, 0} \cdots H_{\text {Meas }, N_{\mathrm{f}}-1}\right]^{\mathrm{T}},
$$

where $[\cdot]^{\mathrm{T}}$ is the transpose operator. Then the corresponding CIR is obtained as

$$
h_{\text {Meas }}(\tau)=\mathbf{p}^{\mathrm{T}}(\tau) \mathbf{H}_{\text {Meas }},
$$

where $\mathbf{p}(\tau)$ is the inverse Fourier transform coefficients as

$$
\mathbf{p}(\tau)=\left[e^{j 2 \pi f_{1} \tau} \cdots e^{j 2 \pi\left(f_{\mathrm{L}}+\left(N_{\mathrm{f}}-1\right) \Delta f\right) \tau}\right]^{\mathrm{T}},
$$

where $f_{\mathrm{L}}$ denotes the lowest measured frequency and $\Delta f$ is the measured frequency step.

In order to improve the calibration accuracy greatly, the temporal deviation in the delay domain should be removed. As a reference to the deterministic part of the channel, only the deterministic propagation paths, including reflection, penetration, diffraction components, are taken into account in RT. The propagation path delay $\tau_{\mathrm{RT}_{\text {det }}, k}$ and the complex amplitude $\eta_{\mathrm{RT}_{\text {det }}, k}$ of the $k$-th deterministic path can be calculated directly by RT. Then the CIR $h_{\mathrm{RT}_{\text {det }}}(\tau)$ at one $\mathrm{Rx}$ location is represented as

$$
h_{\mathrm{RT}_{\mathrm{det}}}(\tau)=\sum_{k=1}^{K} \eta_{\mathrm{RT}_{\mathrm{det}}, k} \delta\left(\tau-\tau_{\mathrm{RT}_{\mathrm{det}}, k}\right),
$$

where $K$ is the total number of the deterministic paths. According to the $k$-th propagation path delay $\tau_{\mathrm{RT}_{\mathrm{det}}, k}$, the corresponding CIRs' interval for the $k$-th path of RT and the measurement is set as $\tau_{\mathrm{RT}_{\text {det }}, k}^{\prime} \in\left[\tau_{\mathrm{RT}_{\text {det }}, k}-5 \cdot \Delta \tau, \tau_{\mathrm{RT}_{\text {det }}, k}+5 \cdot \Delta \tau\right]$, where $\Delta \tau$ is the delay resolution. Furthermore, the crosscorrelation is obtained by

$$
\mathrm{R}_{k}\left(\tau^{\prime}\right)=\int\left|h_{\mathrm{RT}_{\mathrm{det}}}^{*}\left(\tau_{\mathrm{RT}_{\mathrm{det}}, k}^{\prime}\right)\right|\left|h_{\text {Meas }}\left(\tau_{\mathrm{RT}_{\mathrm{det}}, k}^{\prime}+\tau^{\prime}\right)\right| d \tau_{\mathrm{RT}_{\mathrm{det}}, k}^{\prime},
$$

where $|\cdot|$ denotes the absolute value. Then the $k$-th propagation path delay of the measurement $\tau_{\text {Meas }_{\text {det }}, k}$ is obtained by

$$
\tau_{\text {Meas }_{\text {det }}, k} \triangleq \arg \max _{\tau^{\prime}}\left\{R_{k}\left(\tau^{\prime}\right)\right\} .
$$

In addition, the complex amplitude $\eta_{\text {Meas }_{\text {det }}, k}$ of the $k$-th propagation path in the measurement can be obtained as

$$
\eta_{\text {Meas }_{\mathrm{det}}, k}=\frac{\mathbf{p}^{\mathrm{T}}\left(\tau_{\mathrm{Meas}_{\mathrm{det}}, k}\right) \mathbf{H}_{\mathrm{Meas}_{\mathrm{ds}}, k}}{\mathbf{p}^{\mathrm{T}} \mathbf{p}}
$$

where $\mathbf{H}_{\mathrm{Meas}_{\mathrm{ds}}, k}$ is the remaining CTF after subtracting the effect of the $(k-1)$ th deterministic peak as [15]

$$
\mathbf{H}_{\text {Meas }_{\mathrm{ds}}, k}= \begin{cases}\mathbf{H}_{\text {Meas }} & k=1 \\ \mathbf{H}_{\mathrm{Meas}_{\mathrm{ds}}, k-1}-\eta_{\text {Meas }_{\mathrm{det}}, k-1} \mathbf{p}^{*}\left(\tau_{\text {Meas }_{\text {det }}, k-1}\right) & k>1 .\end{cases}
$$

Then the CIRs $h_{\text {Meas det }_{\text {det }}}(\tau)$ and $h_{\text {Meas }_{\mathrm{ds}}}(\tau)$ of the deterministic and diffuse scattering components can be expressed similarly as (9) and (7), respectively. In addition, the CIR of diffuse scattering components based on $\mathrm{RT} h_{\mathrm{RT}_{\mathrm{ds}}}(\tau)$ can be calculated by RT directly only considering the scattering components.

\section{B. MOSA Algorithm}

Simulated annealing (SA) is derived from the physical heating of a material, where the material is critically heated and then gradually cooled until reaching a steady state [5]. It is an iterative optimization algorithm which is able to provide a sub-optimal solution for arbitrary degrees of nonlinearity [6]. In the following, $T$ is a control parameter that corresponds to the temperature in analogy with the physical annealing process and $\mathbf{x}$ is the parameter vector which needs to be optimized. The MOSA algorithm in our presented work uses the weighted sum approach to project the multidimensional parameter space into a one-dimensional space [16]. Only one prior measurement is selected in our current calibration work. The sum-weighted objective function for our case is described as

$$
\mathrm{f}\left(\mathbf{x}, \lambda_{\text {det }}, \lambda_{\text {ds }}\right)=\lambda_{\text {det }} \mathrm{f}_{\text {det }}(\mathbf{x})+\lambda_{\text {ds }} \mathrm{f}_{\text {ds }}(\mathbf{x}),
$$

where the weighting factors should satisfy $\lambda_{\{\cdot\}} \in(0,1)$ and $\lambda_{\operatorname{det}}+\lambda_{\mathrm{ds}}=1$, and the objective function $\mathrm{f}_{\mathrm{det}}(\mathbf{x})$, defined as the root mean square error between the measured and RT simulated tap powers of the deterministic paths, is calculated as

$$
\mathrm{f}_{\mathrm{det}}(\mathbf{x})=\sqrt{\frac{1}{K} \sum_{k=1}^{K}\left(\frac{\left|\eta_{\text {Meas }_{\mathrm{det}}, k}\right|^{2}-\left|\eta_{\mathrm{RT}_{\mathrm{det}}, k}\right|^{2}}{\left|\eta_{\text {Meas }_{\mathrm{det}}, k}\right|^{2}}\right)^{2}}
$$

where $\left|\eta_{\text {Meas }_{\text {det }}, k}\right|$ and $\left|\eta_{\mathrm{RT}_{\text {det }}, k}\right|$ are the amplitudes of the propagation paths normalized by the LOS component, while the objective function $\mathrm{f}_{\mathrm{ds}}(\mathbf{x})$, defined as the mean absolute error (MAE) between the measured and RT simulated instantaneous powers of the diffuse scattering components, is computed as

$$
\mathrm{f}_{\mathrm{ds}}(\mathbf{x})=\frac{1}{N_{\tau}} \int\left|\frac{\left|h_{\mathrm{Meas}_{\mathrm{ds}}, k}\left(\tau_{\mathrm{ex}}\right)\right|^{2}-\left|h_{\mathrm{RT}}, k\left(\tau_{\mathrm{ex}}\right)\right|^{2}}{\left|h_{\mathrm{Meas}_{\mathrm{ds}}, k}\left(\tau_{\mathrm{ex}}\right)\right|^{2}}\right| d \tau_{\mathrm{ex}},
$$

where $N_{\tau}$ is the number of delay points, $\left|h_{\mathrm{Meas}_{\mathrm{ds}}, k}\left(\tau_{\mathrm{ex}}\right)\right|$ and $\left|h_{\mathrm{RT}_{\mathrm{ds}}, k}\left(\tau_{\mathrm{ex}}\right)\right|$ are normalized CIRs defined over the excess 
delay $\tau_{\text {ex }}$. In order to get the optimal parameter vector $\mathbf{x}$, the two objection functions need to be minimized.

The basic steps involved in the MOSA algorithm for RT calibration are illustrated as following:

1) We use the normalized objective functions (15) and (16) that are in the same numerical range, which allows to fix $\lambda_{\mathrm{det}}=0.5$ and $\lambda_{\mathrm{ds}}=0.5$ and avoid another timeconsuming optimization step for these two parameters.

2) Starting with an initial parameter vector $\mathbf{x}^{\prime}$, which is selected from the literature, at an initial temperature $T_{0}$, the solutions of the objective functions $\mathrm{f}_{\mathrm{det}}\left(\mathbf{x}^{\prime}\right)$ and $\mathrm{f}_{\mathrm{ds}}\left(\mathbf{x}^{\prime}\right)$ are evaluated. The corresponding results are put into a Pareto set of solutions. The Pareto set, which is a subset of feasible points of solutions, contains all points in which at least one objective function is minimized.

3) In order to get the realistic results, the ranges of each elements are defined. A new random parameter vector $\mathbf{x}^{\prime \prime}$ is taken from the neighborhood of $\mathbf{x}^{\prime}$ within the given ranges, and the solutions of the related objective functions are re-valuated.

4) Comparing the new solutions with all other solutions in the Pareto set, $\mathbf{x}^{\prime \prime}$ is made as the current parameter vector and the Pareto set is updated if both objective functions are minimized as $\mathrm{f}_{\text {det }}\left(\mathbf{x}^{\prime \prime}\right) \leq \mathrm{f}_{\text {det }}\left(\mathbf{x}^{\prime}\right)$ and $\mathrm{f}_{\mathrm{ds}}\left(\mathbf{x}^{\prime \prime}\right) \leq \mathrm{f}_{\mathrm{ds}}\left(\mathbf{x}^{\prime}\right)$. Then the process restarts from step 6 . If $\mathbf{x}^{\prime \prime}$ is not the optimal parameter vector for both objection functions, then

$$
\Delta \mathrm{f}=\mathrm{f}\left(\mathbf{x}^{\prime \prime}, \lambda_{\text {det }}, \lambda_{\mathrm{ds}}\right)-\mathrm{f}\left(\mathbf{x}^{\prime}, \lambda_{\text {det }}, \lambda_{\mathrm{ds}}\right)
$$

is evaluated.

5) In order to avoid being trapped into a local minimum, the parameter vector $\mathbf{x}^{\prime \prime}$ is accepted as the current parameter vector with the probability

$$
p= \begin{cases}e^{\frac{\Delta \mathrm{f}}{T}} & \text { if } \Delta \mathrm{f}>0 \\ 1 & \text { if } \Delta \mathrm{f} \leq 0 .\end{cases}
$$

If $\mathbf{x}^{\prime \prime}$ is accepted, $\mathbf{x}^{\prime \prime}$ is made as the current parameter vector. If $\mathbf{x}^{\prime \prime}$ is not accepted, $\mathbf{x}^{\prime}$ is retained as the current parameter vector.

6) The algorithm restarts running by selecting the referenced solutions of the objective functions corresponding to the minimum $\mathrm{f}\left(\mathbf{x}, \lambda_{\mathrm{det}}, \lambda_{\mathrm{ds}}\right)$ in the Pareto set.

7) The mentioned steps repeatedly run $L$ times at the same temperature $T$. Then the algorithm restarts with the initial temperature $T_{0}$ reduced by a factor $N_{\mathrm{T}}$, where $N_{\mathrm{T}}<1$.

8) The aforementioned steps run iteratively until the predefined number of iterations $M$ is achieved.

\section{Measurement CAmpaign and RT Setup}

A 2-D top view of the indoor scenario used for both the measurement campaign and RT is illustrated in Fig.1, where the different materials are sketched with different colors. The Rhode \& Schwarz ZVA-24 vector network analyzer (VNA) was used to measure the frequency-domain UWB channel at $N_{\mathrm{f}}=7501$ frequency points over the frequency range from $f_{\mathrm{L}}=3.1 \mathrm{GHz}$ to $f_{\mathrm{H}}=10.6 \mathrm{GHz}$ in the Signal Processing and Speech Communication laboratory at Graz University of Technology [4]. The significant blocks are concrete walls, glass windows and metal pillars [17].

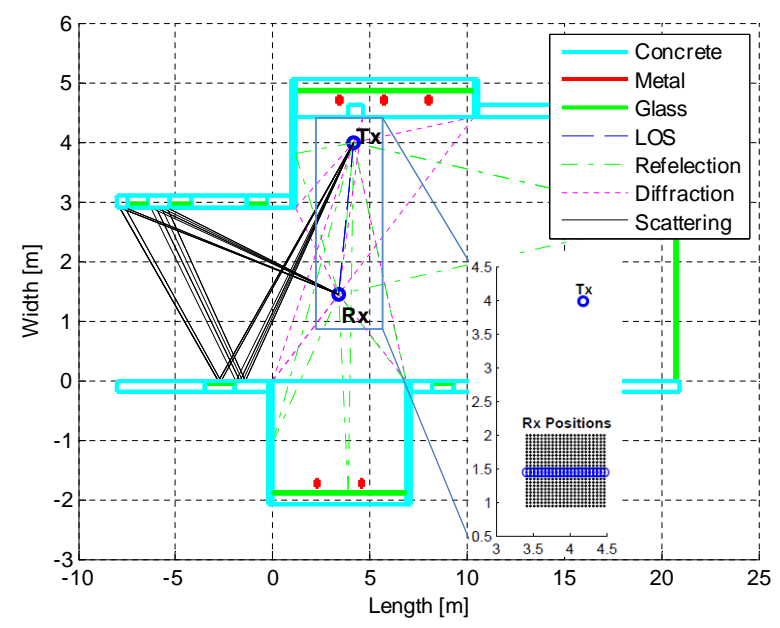

Fig. 1. 2-D top view of the indoor scenario including a close-up view of the grid Rx's positions together with 22 positions marked in blue line where low-complexity sub-band divided RT simulations are implemented.

Low-complexity sub-band divided RT simulations are computed for the middle horizontal line of the grid highlighted in blue in Fig. 1. The measurements at the middle point of the horizontal line is used for the calibration. The Tx and $\mathrm{Rx}$ antennas in the RT simulation are dipole antennas corresponding to the antennas used in measurements. In addition, the entire UWB bandwidth $B=7.5 \mathrm{GHz}$ is divided into $I=15$ subbands with $B_{i}=500 \mathrm{MHz}$ each. The optimized material parameters are the elements of the parameter vector $\mathbf{x}$

$$
\mathbf{x}=\left[\varepsilon_{\mathbf{c}, r}, \sigma_{\mathbf{c}}, S_{\mathbf{c}}, \alpha_{\mathbf{c}}, \varepsilon_{\mathbf{g}, r}, S_{\mathbf{g}}, \alpha_{\mathbf{g}}\right],
$$

where $\mathbf{c}$ indicates concrete and $\mathbf{g}$ glass. The metallic block is considered as a perfect electric conductor. The conductivity of glass is $\sigma_{\mathbf{c}}=0 \mathrm{~S} / \mathrm{m}$. The involved propagation mechanisms for the calibration are LOS, reflection up to the third order, penetration, single diffraction, single bounce scattering, and scattering-reflection cases.

\section{Numerical Results}

\section{A. Deterministic and Diffuse Scattering Paths Separation from Measurements}

Based on (10), the deterministic paths in the measurements are identified such that slight time of arrival deviations of the deterministic paths can be tolerated. The precise mapping of the measured and RT simulated propagation paths can help to improve the calibration accuracy. Fig. 2 shows the normalized amplitude $\left|\eta_{\text {det }}\right|$ of the deterministic paths in measurements and low-complexity sub-band divided RT before the calibration. In order to avoid a large deviation of the power of detected paths, a threshold of the difference between $\left|\eta_{\text {Meas }_{\text {det }}, k}\right|$ and $\left|\eta_{\mathrm{RT}_{\mathrm{det}}, k}\right|$ of the $k$-th deterministic propagation path is set to $20 \mathrm{~dB}$. If the difference exceeds $20 \mathrm{~dB}$, the $k$-th deterministic path is deleted. Removing the deterministic components from the 


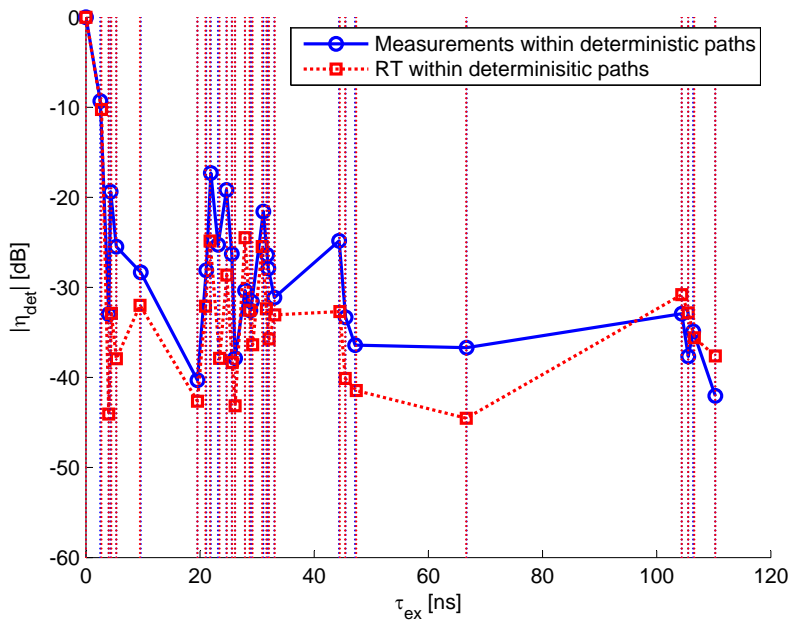

Fig. 2. Normalized amplitude $\left|\eta_{\text {det }}\right|$ of the deterministic paths in measurements and low-complexity sub-band divided RT before the calibration.

measurements, the remaining diffuse scattering components are calculated as the inverse Fourier transform of $\mathbf{H}_{\mathrm{Meas}_{\mathrm{ds}}, k}$. The CIRs $\left|h_{\text {Meas }_{\mathrm{ds}}}\left(\tau_{\mathrm{ex}}\right)\right|$ and $\left|h_{\mathrm{RT}_{\mathrm{ds}}}\left(\tau_{\mathrm{ex}}\right)\right|$ are shown in Fig. 3.

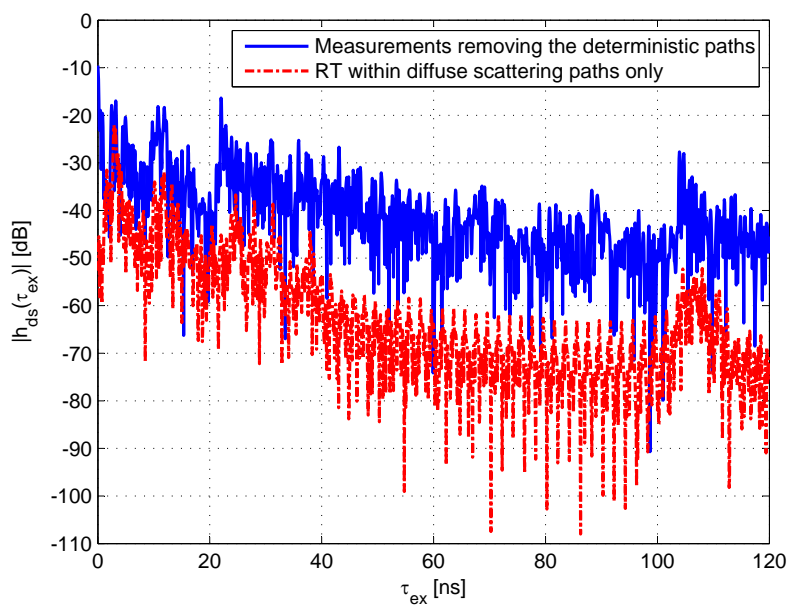

Fig. 3. Normalized CIRs $\left|h_{\mathrm{ds}}\left(\tau_{\mathrm{ex}}\right)\right|$ of the measurements and the lowcomplexity sub-band divided RT before the calibration considering the diffuse scattering paths only.

\section{B. Calibration Results}

The initial parameter vector is picked up from [4] as

$$
\mathbf{x}^{\prime}=[6,0.08,0.4,4,5.5,0.4,4] .
$$

Each element of $\mathbf{x}$ has its individual range, which are summarized from [3], [18]-[22], as

$$
\left\{\begin{array}{l}
4 \leq \varepsilon_{\mathbf{c}, r} \leq 9 \\
0.023 \leq \sigma_{\mathbf{c}} \leq 0.5 \\
0 \leq S_{\mathbf{c}} \leq 1 \\
1 \leq \alpha_{\mathbf{c}} \leq 4 \\
2 \leq \varepsilon_{\mathbf{g}, r} \leq 8 \\
0 \leq S_{\mathbf{g}} \leq 1 \\
1 \leq \alpha_{\mathbf{g}} \leq 4
\end{array}\right.
$$

In order to avoid the random phases of the scattering components in RT influencing the $\mathrm{f}_{\mathrm{ds}}(\mathbf{x})$, a matrix with $I \times N_{\mathrm{s}}$ random elements is given at the beginning of the optimization process, where the number of scattering paths calculated from RT is $N_{s}=26614$. The control factors in calibration is set as $\left[T, N_{\mathrm{T}}, L, M\right]=[2,0.95,20,5000]$. In Fig. 4, the solutions of

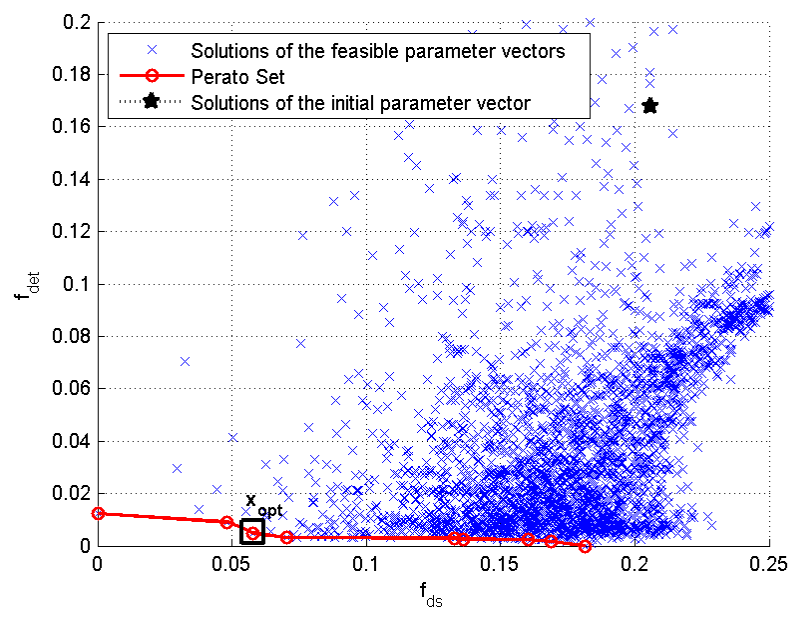

Fig. 4. Solutions of objective functions related to the feasible parameter vectors within the Pareto front.

objective functions related to the feasible parameter vectors are shown, and the Pareto front is given. In order to make the optimized results clear, the obtained $\mathrm{f}_{\mathrm{det}}(\mathbf{x})$ and $\mathrm{f}_{\mathrm{ds}}(\mathbf{x})$ are normalized by the respective minimum values. Taking the solutions in the Pareto set into consideration, one parameter vector $\mathbf{x}_{\mathrm{opt}}$ is selected

$$
\mathbf{x}_{\text {opt }}=[8.92,0.046,0.74,3,3.7,0.32,3] .
$$

It can be seen that $S_{\mathbf{c}}$ becomes larger, while $S_{\mathbf{g}}$ becomes smaller, which is confirmed by reality that the surface of the concrete is relatively rougher and of the glass is relatively smoother. Moreover, the smaller $\alpha_{\mathbf{c}}$ and $\alpha_{\mathbf{g}}$ indicate the a widening of the scattering lobe.

\section{PDP and RMS Delay Spread Comparison}

Using the parameter vector $\mathbf{x}_{\mathrm{opt}}$ in low-complexity sub-band divided RT, the normalized PDPs and the RMS delay spread $\tau_{\text {rms }}$ are compared by averaging the normalized CIRs over the horizontal 22 positions, $\mathrm{Rx}_{\mathrm{idx}}$. The results are shown in Fig. 5. It is worth mentioning that the reflection-scattering case is also considered by RT. Based on the optimized material parameters, the difference of normalized PDPs between the measurements and RT simulation is reduced by $10 \mathrm{~dB}$ and the RMS delay spread is improved about 5ns despite only one prior measurement is used for the calibration. Moreover, it can be seen that the power of the diffuse components is increased, while the power of the deterministic components are not influenced. The reasons for the remaining gap are the large dimensions of the environment which cannot be modeled absolutely accurate and the higher-order propagation mechanisms that are not considered by our RT algorithm. 

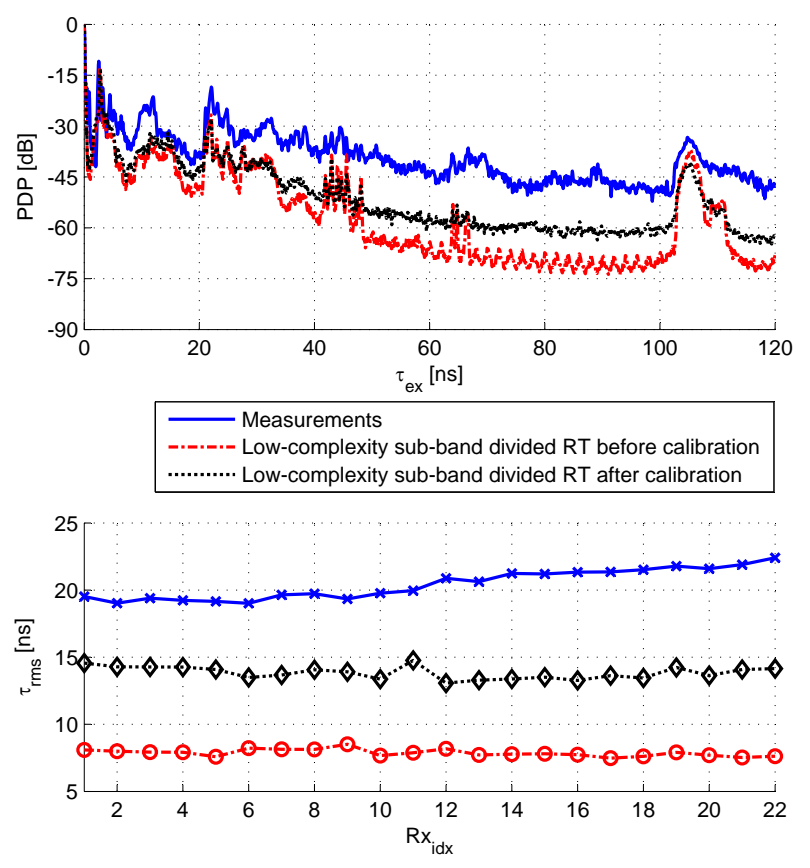

Fig. 5. Normalized PDP and RMS delay spread comparison based on the measurements, low-complexity sub-band divided RT before calibration, and low-complexity sub-band divided after calibration.

\section{CONCLUSiON}

In this paper, we calibrated the sub-band divided RT algorithm for UWB indoor channels using the MOSA algorithm to optimize the dielectric material parameters and the scattering parameters. Our method allows the joint tuning of these parameters to reduce the mismatch between measurements and RT simulations. Firstly, a low-complexity sub-band divided RT algorithm is implemented to reduce the computational complexity. Then the deterministic and diffuse scattering paths are distinguished in measurement data relying upon cross correlation and search and subtract algorithm. The MOSA algorithm is based on the weighted sum approach, in which two objective functions are used. From the finally determined Pareto set, an optimal set of material parameters can be obtained. Based on the optimal material parameters, the differences of the normalized PDPs and the RMS delay spread between measurements and low-complexity sub-band divided RT simulation results are minimized.

\section{REFERENCES}

[1] B. Alavi, N. Alsindi, and K. Pahlavan, "UWB channel measurements for accurate indoor localization," in Military Communications Conference, 2006. MILCOM 2006. IEEE, 2006, pp. 1-7.

[2] P. Meissner and K. Witrisal, "Analysis of position-related information in measured UWB indoor channels," in Antennas and Propagation (EUCAP), 2012 6th European Conference on, 2012, pp. 6-10.

[3] V. Degli-Esposti, F. Fuschini, E. M. Vitucci, and G. Falciasecca, "Measurement and modelling of scattering from buildings," Antennas and Propagation, IEEE Transactions on, vol. 55, no. 1, pp. 143 - 153, Jan. 2007.

[4] P. Meissner, M. Gan, F. Mani, E. Leitinger, M. Froehle, C. Oestges, T. Zemen, and K. Witrisal, "On the use of ray tracing for performance prediction of UWB indoor localization systems," in IEEE ICC 2013
Workshop on Advances in Network Localization and Navigation (ANLN), June 2013

[5] J. Jemai, P. C. F. Eggers, G. Pedersen, and T. Kurner, "Calibration of a UWB sub-band channel model using simulated annealing," Antennas and Propagation, IEEE Transactions on, vol. 57, no. 10, pp. 3439-3443, 2009.

[6] S. Priebe, M. Jacob, and T. Kurner, "Calibrated broadband ray tracing for the simulation of wave propagation in $\mathrm{mm}$ and sub-mm wave indoor communication channels," European Wireless, 2012. EW. 18th European Wireless Conference, pp. 1 - 10, April 2012.

[7] M. Gan, P. Meissner, F. Mani, E. Leitinger, M. Froehle, C. Oestges, K. Witrisal, and T. Zemen, "Low-complexity sub-band divided ray tracing for UWB indoor channel," in IEEE Wireless Communications and Networking Conference (WCNC), April 2014, submitted.

[8] J. Jemai and T. Kürner, "Towards a performance boundary in calibrating indoor ray tracing models," EURASIP J. Wireless Comm. and Networking, vol. 2009, 2009.

[9] C. Oestges, B. Clerckx, L. Raynaud, and D. Vanhoenacker-Janvier "Deterministic channel modeling and performance simulation of microcellular wide-band communication systems," Vehicular Technology, IEEE Transactions on, vol. 51, no. 6, pp. 1422 - 1430, Nov. 2002.

[10] F. Mani and C. Oestges, "Evaluation of diffuse scattering contribution for delay spread and crosspolarization ratio prediction in an indoor scenario," in Antennas and Propagation (EuCAP), 2010 Proceedings of the Fourth European Conference on, April 2010, pp. 1 - 4.

[11] F. Mani, F. Quitin, and C. Oestges, "Accuracy of depolarization and delay spread predictions using advanced ray-based modeling in indoor scenarios," EURASIP Jounal in Wireless Commmunications and Networking, vol. 2011, p. 11, 2011.

[12] J. Jemai, P. Eggers, G. Pedersen, and T. Kürner, "On the applicability of deterministic modelling to indoor UWB channels," in Proceedings of the 3rd Workshop on Positioning, Navigation and Communication (WPNC'06), 2006.

[13] H. Sugahara, Y. Watanabe, T. Ono, K. Okanoue, and S. Yarnazaki, "Development and experimental evaluations of "RS-2000" - a propagation simulator for UWB systems," in Ultra Wideband Systems, 2004. Joint with Conference on Ultrawideband Systems and Technologies. Joint UWBST IWUWBS. 2004 International Workshop on, May 2004, pp. 76 -80 .

[14] G. Tiberi, S. Bertini, W. Malik, A. Monorchio, D. Edwards, and G. Manara, "Analysis of realistic ultrawideband indoor communication channels by using an efficient ray-tracing based method," Antennas and Propagation, IEEE Transactions on, vol. 57, no. 3, pp. 777 - 785, Mar. 2009.

[15] T. Santos, J. Karedal, P. Almers, F. Tufvesson, and A. Molisch, "Modeling the ultra-wideband outdoor channel: Measurements and parameter extraction method," Wireless Communications, IEEE Transactions on, vol. 9, no. 1, pp. 282-290, 2010.

[16] B. Suman and P. Kumar, "A survey of simulated annealing as a tool for single and multiobjective optimization," Journal of the Operational Research Society, vol. 57, no. 10, pp. 1143-1160, 2005. [Online]. Available: http://dx.doi.org/10.1057/palgrave.jors.2602068

[17] P. Meissner, E. Leitinger, M. Froehle, and K. Witrisal. (2013) MeasureMINT. [Online]. Available: http://www.spsc.tugraz.at/tools/ UWBmeasurements

[18] A. Muqaibel, A. Safaai-Jazi, A. Bayram, A. M. Attiya, and S. Riad, "Ultrawideband through-the-wall propagation," Microwaves, Antennas and Propagation, IEE Proceedings, pp. 581-588, 2005.

[19] B. De Backer, H. Borjeson, D. De Zutter, and F. Olyslager, "Propagation mechanisms for uhf wave transmission through walls and windows," Vehicular Technology, IEEE Transactions on, vol. 52, no. 5, pp. 12971307, 2003.

[20] H. Suzuki and A. Mohan, "Measurement and prediction of high spatial resolution indoor radio channel characteristic map," Vehicular Technology, IEEE Transactions on, vol. 49, no. 4, pp. 1321-1333, 2000.

[21] R. Dalke, C. Holloway, P. McKenna, M. Johansson, and A. Ali, "Effects of reinforced concrete structures on rf communications," Electromagnetic Compatibility, IEEE Transactions on, vol. 42, no. 4, pp. 486-496, 2000.

[22] J. Jemai, T. Krner, A. Varone, and J. F. Wagen, "Determination of the permittivity of building materials through wlan measurements at 2.4 ghz," in Personal, Indoor and Mobile Radio Communications, 2005. PIMRC 2005. IEEE 16th International Symposium on, vol. 1, 2005, pp. $589-593$. 